\title{
Quantification of graphene based core/shell quantum dots from first principles
}

\author{
X. Y. Cui, ${ }^{1, a)}$ R. K. Zheng, ${ }^{1}$ Z. W. Liu, ${ }^{1}$ L. Li,${ }^{1}$ C. Stampfl, ${ }^{2}$ and S. P. Ringer ${ }^{1}$ \\ ${ }^{1}$ Australian Centre for Microscopy and Microanalysis, The University of Sydney, New South Wales 2006, \\ Australia \\ ${ }^{2}$ School of Physics, The University of Sydney, New South Wales 2006, Australia
}

(Received 19 August 2011; accepted 7 October 2011; published online 31 October 2011)

\begin{abstract}
Density functional calculations are performed to study the electronic structure of recently proposed graphene/graphane based core/shell quantum dots, which have a type I band alignment and exhibit quantized carrier energy levels. Strong confinement is robust with shell thickness. The bandgap, band offset, and the number of confined carrier orbitals with different size and geometry are determined. Our findings indicate that these core/shell dots are potentially well suited for the design of advanced diode lasers and room-temperature single electron devices. The proposed method to determine the number of confined orbitals is applicable for other quantum dot systems. (C) 2011 American Institute of Physics. [doi:10.1063/1.3657488]
\end{abstract}

Graphene-based quantum dots (GQDs) exhibit unique properties in connection with quantum confinement effects and are currently of intense scientific and technological interest. The electronic structures and hence properties and functionalities can be tailored by size and shape, affording the possibility of important developments, including spin qubits, ${ }^{1}$ single electron transistors, ${ }^{2,3}$ photovoltaics, ${ }^{4-6}$ and light-emitting diodes. ${ }^{7-9}$

For the most studied bare (without shell) GQDs and graphene ribbons, the optimization of device performance requires a size less than $10 \mathrm{~nm}$ with smooth edges (i.e., the boundary roughness is in the order of $1 \mathrm{~nm}$ to achieve specular scatterings ${ }^{10}$ ), which has been proved challenging to achieve experimentally. Alternatively, the hybrid core/shell structured QDs are often chemically and thermally more stable than the corresponding bare dots. Moreover, because the shell can serve as an additional confinement potential for the carriers, core/shell QDs can exhibit stronger fluorescence quantum yield. ${ }^{11}$

Recently, Singh et al., ${ }^{12}$ proposed that extraction of hydrogen from graphane ${ }^{13,14}$ (hydrogenated graphene) naturally forms graphene/graphane core/shell QDs, in which the carriers are highly contained. It is expected that the realization of such heterostructure will yield intriguing phenomena as those in bare GQDs, as well the development of new electronic and optoelectronic devices. However, to fulfill these perspectives, it is crucial to study the electronic structure of these nanostructures, in particular, to unravel the carrier energy levels and the band alignment, as well as their correlation with the size and geometry of both core and shell regions. These targets forge the main motivation of this paper.

Density functional theory (DFT) calculations are capable to characterize the aforementioned properties. ${ }^{15}$ The common challenge in QD simulation using DFT rests with a multitude of length scales involved and the resulting size of the system. In the present study, several factors enable a full first principles study of practical sized GQDs based on DFT: (i) the 2-dimensional geometry involved; (ii) light elements, $\mathrm{H}$ and

\footnotetext{
${ }^{\text {a) }}$ Author to whom correspondence should be addressed. Electronic mail: carl.cui@sydney.edu.au.
}

C; (iii) the small electron Fermi wavelength of graphene, namely $0.74 \mathrm{~nm} .{ }^{10}$ According to Ref. 12, QDs with hexagonal structure with sharp interface between graphene and graphane are energetically stable. We therefore construct various QDs with diameters of core region ranging $0.3 \sim 1.5 \mathrm{~nm}$, as shown in Fig. 1. Single layer graphene/graphane QDs were calculated using large supercells of 576 atoms where the corresponding lattice constants $a=30.52 \AA, \quad b=26.42 \AA$, $c=29.62 \AA$. Supercells of 360 atoms and 256 atoms were also used to study the effect of shell-thickness. We perform all-electron DFT calculations using the generalized gradient approximation ${ }^{16}$ with the $\mathrm{DMol}^{3}$ code. ${ }^{17}$ The wave functions are expanded in terms of a double-numerical quality basis set with a real-space cutoff of 9 bohr. Reciprocal space k-point meshes of $1 \times 2 \times 1$ mesh were employed. We allowed full atomic relaxation, where the forces on the atoms were less than $0.005 \mathrm{eV} / \AA$.

Hydrogenation of graphene to form graphane ${ }^{13,14}$ changes the hybridization of carbon atoms from $s p^{2}$ to $s p^{3}$, and from a semimetal (bandgap $=0 \mathrm{eV}$ ) to an insulator (DFT: direct bandgap of $3.9 \mathrm{eV}$, compared to $3.7 \mathrm{eV}$ in Ref. 13). The calculated lattice parameters of graphene and graphane are $2.47 \AA$ and $2.52 \AA$, respectively. Embedding graphene islands into graphane matrix, or more preciously, removing $\mathrm{H}$ atoms from both sides of graphane lead to structural distortion mainly around the boundary regions, whereas the central $\mathrm{C}$ atoms tend to form flat graphene sheet.

In QDs, charge carriers are confined in all three spatial dimensions; and because of this, the energy spectra of electrons and holes can be considered discrete. This offers a wide range of effective band-gap (optical emission) tunability of both interband transitions and improved intersubband transitions. The total density of state (DOS) of the QDs are shown in Fig. 2(a), where the quantization of the energy levels can be clearly seen. The bandgap values, defined as the energy difference between the highest occupied molecular orbital (HOMO) and lowest unoccupied molecular orbital (LUMO) states, are shown in Table I. The bandgap values, corresponding to interband emission for optical applications, depend strongly on the size of the QDs, ranging from 

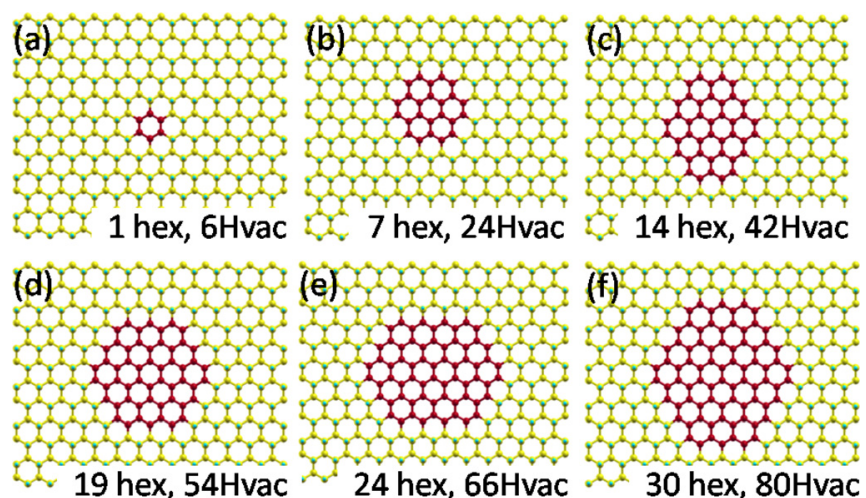

FIG. 1. (Color online) The graphene/graphane core/shell based quantum dots structures. In the core (graphene) region, carbon atoms are shown as (dark) red, in shell (graphane) region as (light) yellow, and $\mathrm{H}$, as green spheres. The number of hexagons ("hex") and the number of removed $\mathrm{H}$ atoms, or hydrogen vacancy ("Hvac") at the core regions are listed.

$3.97 \mathrm{eV}$ to $0.98 \mathrm{eV}$, well covering the visible light range $3.1 \mathrm{eV}$ to $1.7 \mathrm{eV}$. This shows that such QDs could potentially be engineered for active visible light sources. As expected, in general, smaller QDs correspond to larger bandgap values due to a stronger quantum confinement effect. We note that the bandgap values also depend on the shape of the quantum dots; for instance, the smaller $42 \mathrm{Hvac}$ configuration has a smaller HOMO-LUMO separation $(1.67 \mathrm{eV})$ than that of the $54 \mathrm{Hvac}(1.74 \mathrm{eV})$. Another feature is that for a given QD, the bandgap values slightly vary as a function of the shell thickness, namely, thicker shell produce smaller bandgap. Here there are two competing factors in affecting the

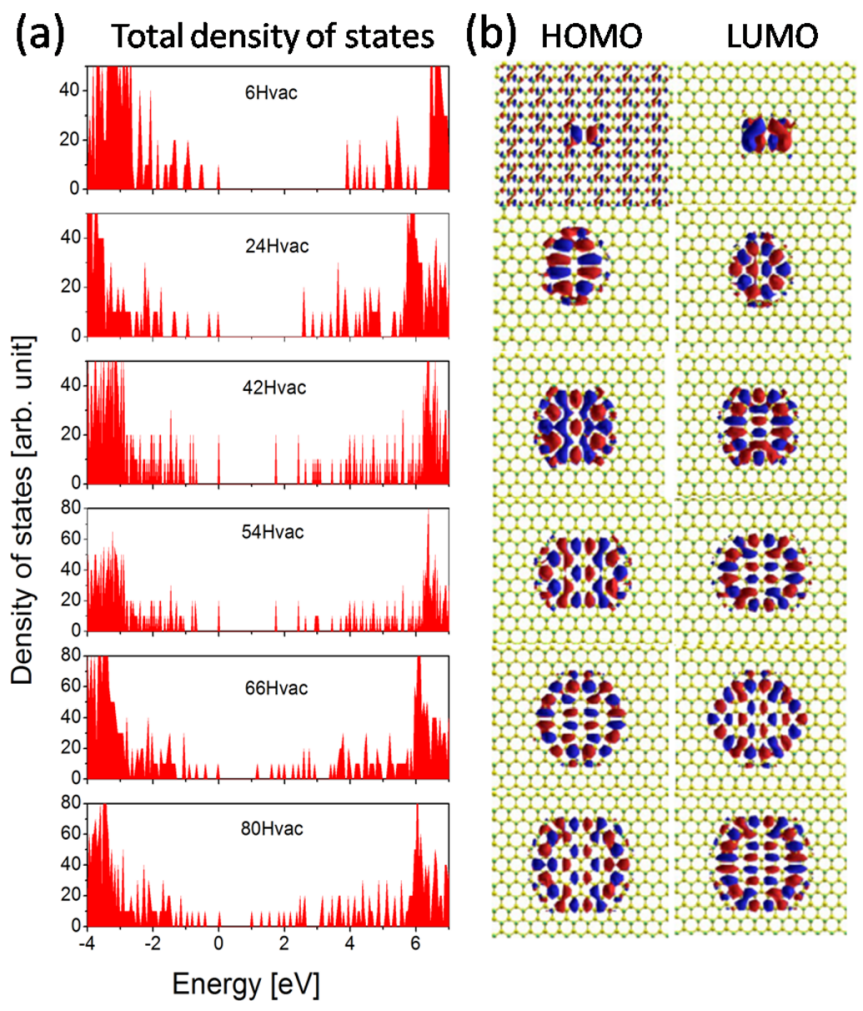

FIG. 2. (Color online) (a) Total DOS showing the quantization of the energy spectrum and, (b) the HOMO and LUMO for various graphene/graphane core/shell GQDs. Note the magnitude of DOS indicates the degree of state degeneracy.
TABLE I. DFT calculated energy bandgap (in eV) and natural valence band offset (in eV) for various QDs using the different sized supercells (576-, 360-, and 256-atom).

\begin{tabular}{lccccccc}
\hline \hline & \multicolumn{3}{c}{ Energy bandgap } & & \multicolumn{3}{c}{ Valence band offset } \\
\cline { 2 - 4 } \cline { 7 - 8 } Dot Config. & $576-$ & $360-$ & $256-$ & & $576-$ & $360-$ & $256-$ \\
\hline 6 Hvac & 3.967 & 3.973 & 3.973 & & 1.676 & 1.676 & 1.681 \\
$24 \mathrm{Hvac}$ & 2.596 & 2.602 & 2.607 & & 1.426 & 1.449 & 1.462 \\
$42 \mathrm{Hvac}$ & 1.667 & 1.669 & - & & 1.336 & 1.355 & - \\
$54 \mathrm{Hvac}$ & 1.736 & 1.741 & - & & 1.281 & 1.318 & - \\
$66 \mathrm{Hvac}$ & 1.197 & - & - & & 1.256 & - & - \\
$80 \mathrm{Hvac}$ & 0.979 & - & - & & 1.231 & - & - \\
\hline \hline
\end{tabular}

bandgap: one is for an isolated dot, the thicker shell exhibits stronger confinement, and thus larger bandgap; the other factor lies in the interaction between the QDs as simulated here using periodic supercells. Our results suggest that the latter factor tends to decrease the bandgap. Overall, the shellthickness dependence of the bandgap in graphene/graphane QDs is much weaker than that of III-Nitride quantum well structures, ${ }^{18,19}$ and also of that for GQDs embedded in hexagonal $\mathrm{BN},{ }^{20}$ indicating a strong confinement even using rather thin shells.

For possible QD-based laser applications, it is important to study the nature of the discrete states (confined or extended) and to determine the number of confined carrier orbitals. We have calculated the spatial distribution of the magnitude of the wave functions of the near-edge states for all the QDs. In Fig. 2(b), we show such quantities for the HOMO and LUMO states for the QDs using the 576-atomsupercell. The same procedure was applied to other near-edge states to determine the nature of orbitals. For each orbital, we have considered the state degeneracy into account. In such a way we obtain the number of confined carrier (electron and hole) orbitals, which are listed in Table II. As the QD size increases, more carrier (both electron and hole) orbitals, are confined in the core region; consequently, the adjacent carrier level spacing/separation becomes smaller. All the QDs show strong confinement, except for the 6 Hvac structure, in which only 2 hole-states are well confined in the core (graphene) region. For the first electron state (HOMO), there is substantial charge leakage to graphane matrix. So the $6 \mathrm{Hvac}$ dot can be regarded as the lower size limit of graphene/graphene core/shell QDs. While experimentally counting of confined carrier orbitals in QDs is not a straightforward task, our proposed theoretical approach is believed to be applicable to other quantum dots. For laser applications, the all-direction confinement in QDs results in superior lasing characteristics including lower threshold currents and improved temperature

TABLE II. Number of confined occupied (electron), $N_{e}$, and unoccupied (hole), $N_{h}$, orbitals in the various QDs. Note here each orbital contains two carriers.

\begin{tabular}{lcccccc}
\hline \hline Dot Config. & $6 \mathrm{Hvac}$ & $24 \mathrm{Hvac}$ & $42 \mathrm{Hvac}$ & $54 \mathrm{Hvac}$ & $66 \mathrm{Hvac}$ & $80 \mathrm{Hvac}$ \\
\hline$N_{e}$ & 0 & 2 & 3 & 6 & 6 & 8 \\
$N_{h}$ & 3 & 5 & 8 & 12 & 14 & 17 \\
\hline \hline
\end{tabular}


stability. $^{21,22}$ The well-separated carrier levels, much larger than the thermal energy, (i.e., $\sim 24 \mathrm{meV}$ for room temperature), indicate that these GQDs could potentially be used for high-performance laser applications.

The band alignment of graphene/graphane is a so-called type-I heterostructure, in which both electrons and holes are confined in the core region. We calculate the natural valence band offset (VBO) (Ref. 23) as $\Delta E_{V B O}=E_{\text {Graphene }}^{b}-E_{\text {Graphene }}^{b}$ $-\Delta E_{B}$. Here the bulk terms, $E_{\text {Graphene }}^{b}$ and $E_{\text {Graphene, }}^{b}$ correspond to the binding energy difference between the core-level (here we use C- $1 s$ orbital) and the HOMO for pure graphene and graphane, and the interface term, $\Delta E_{B}$, is the binding energy difference for the core-level C-1s state in the core and shell regions of the QDs. More details can be found in Ref. 19. The obtained VBOs for all QDs are listed in Table I. It can be seen that smaller QDs possess larger VBO values regardless of the supercell sizes; and for a given sized QD, separation (using larger supercell) tends to decrease this value. Since the bulk term is a constant for all the QDs, this variation originates purely from the interface term. Our results suggest that for smaller QDs, thus stronger confinement, lead to larger VBOs. In analogy to InN/GaN quantum well systems, ${ }^{19}$ our results reveal a possible route for band alignment engineering by controlling the size of QDs.

Due to the intrinsic long coherence times, in combination with fast operating times, graphene-based QDs are envisioned as possible building blocks for future quantum information processors. ${ }^{1}$ Devices that confine and process single electrons represent an important scaling limit of electronics, ${ }^{24}$ such as single electron transistors (SETs) with high on-off ratios. The performance of SETs is based on the Coulomb blockade effect, ${ }^{25}$ which is a manifestation of discrete carrier energy levels in QDs. To realize room-temperature operation, a well-separated carrier energy spectrum is indispensible. Based on our results, we argue that graphene/ graphane core/shell QDs are promising candidates in this regard. The size and shape of these structures, and therefore the number of electrons they contain, can be precisely controlled. Another appealing feature is that strong confinement can be obtained using a thin barrier which is desirable for efficient tunneling transport to facilitate SET operation.

We acknowledge the computing resources provided by the National Computational Infrastructure (Australia) and support from the Australian Research Council. Support from AMMRF node at the University of Sydney (ACMM) is gratefully acknowledged.

${ }^{1}$ B. Trauzettel, D. V. Bulaev, D. Loss, and G. Burkard, Nat. Phys. 3, 192 (2007).

${ }^{2}$ L. A. Ponomarenko, F. Schedin, M. I. Katsnelson, R. Yang, E. W. Hill, K. S. Novoselov, and A. K. Geim, Science 320, 356 (2008).

${ }^{3}$ C. Stampfer, J. Gttinger, F. Molitor, D. Graf, T. Ihn, and K. Ensslin, Appl. Phys. Lett. 92, 012102 (2008).

${ }^{4}$ X. Yan, X. Cui, B. S. Li, and L. S. Li, Nano Lett. 10, 1869 (2010).

${ }^{5}$ P. V. Kamat, J. Phys. Chem. Lett. 2, 242 (2011).

${ }^{6}$ L. Mueller, X. Yan, B. Dragnea, and L.-S. Li, Nano Lett. 11, 56 (2011).

${ }^{7}$ D. Y. Pan, J. C. Zhang, Z. Li, and M. H. Wu, Adv. Mater. 22, 734 (2010).

${ }^{8}$ X. Yan, X. Cui, and L.-S. Li, J. Am. Chem. Soc. 132, 5944 (2010).

${ }^{9}$ J. Shen, Y. Zhu, C. Chen, X. Yang, and C. Li, Chem. Commun. 47, 2580 (2011).

${ }^{10}$ L. Tapaszto, G. Dobrik, P. Nemes-Incze, G. Vertesy, Ph. Lambin, and L. P. Biro, Phys. Rev. B 78, 233407 (2008).

${ }^{11}$ L. Dworak, V. V. Matylitsky, V. V. Breus, M. Braun, T. Basche, and J. Wachtveitl, J. Phys. Chem. C, 115, 3949 (2011).

${ }^{12}$ K. Singh, E. S. Penev, and B. I. Yakobson, ACS Nano 4, 3510 (2010).

${ }^{13}$ J. O. Sofo, A. S. Chaudhari, and G. D. Barber, Phys. Rev. B 75, 153401 (2007).

${ }^{14}$ D. C. Elias, R. R. Nair, T. M. G. Mohiuddin, S. V. Morozov, P. Blake, M. P. Halsall, A. C. Ferrari, D. W. Boukhvalov, M. I. Katsnelson, A. K. Geim, and K. S. Novoselov, Science 323, 610 (2009).

${ }^{15} \mathrm{~J}-\mathrm{B}$ Li and L-W Wang, Appl. Phys. Lett. 84, 3648 (2004).

${ }^{16}$ J. P. Perdew, K. Burke, and M. Ernzerhof, Phys. Rev. Lett. 77, 3865 (1996).

${ }^{17}$ B. Delley, J. Chem. Phys. 113, 7756 (2000); J. Chem. Phys. 92, 508 (1990).

${ }^{18}$ X. Y. Cui, D. J. Carter, M. Fuchs, B. Delley, S. H. Wei, A. J. Freeman, and C. Stampfl, Phys. Rev. B 81, 155301 (2010).

${ }^{19}$ C. C. Shieh, X. Y. Cui, B. Delley, and C. Stampfl, J. Appl. Phys. 109, 083721 (2011).

${ }^{20}$ J. W. Li and V. B. Shenoy, Appl. Phys. Lett. 98, 013105 (2011).

${ }^{21}$ Y. Arakawa and H. Sakaki, Appl. Phys. Lett. 40, 939 (1982).

${ }^{22}$ A. J. Shields, Nat. Photonics, 1, 215 (2007).

${ }^{23}$ S. H. Wei and A. Zunger, Appl. Phys. Lett. 69, 2719 (1996).

${ }^{24}$ R. Hanson, L. P. Kouwenhoven, J. R. Petta, S. Tarucha, and L. M. K. Vandersypen, Rev. Mod. Phys. 79, 1217 (2007).

${ }^{25}$ T. A. Fulton and G. J. Dolan, Phys. Rev. Lett. 59, 109 (1987). 